\title{
Erythema Migrans
}

National Cancer Institute

\section{Source}

National Cancer Institute. Erythema Migrans. NCI Thesaurus. Code C119047.

A red, concentrically-expanding rash sometimes with central clearing, that is part of the first stage of Lyme disease caused by infection with Borrelia species. 